\title{
Control of phase shifting transformers by multiple transmission system operators
}

\author{
A. Marinakis M. Glavic Senior Member, IEEE T. Van Cutsem, Fellow, IEEE
}

\begin{abstract}
A general framework is proposed for the control of phase shifting transformers owned by several TSOs, taking into account their interactions. The proposed solution is the Nash equilibrium of a sequence of optimizations performed by the various TSOs, each of them taking into account the other TSOs' control settings as well as operating constraints relative to the whole system. The method is applied to a linearized network model and illustrated on the IEEE 118-bus system.
\end{abstract}

Index Terms -Phase shifting transformers, multi-objective optimization, constrained optimization, Nash equilibrium.

\section{INTRODUCTION}

The Phase Shifting Transformer (PST) offers the opportunity to partially control the flows in a power system. It is one of the principal control devices used to direct power flows in specific parts of the transmission network. Although the operation of PSTs incurs maintenance costs and losses, it remains less costly than generation rescheduling and definitely preferred to load shedding [1].

Coordinated control of multiple phase shifters in a grid may alleviate congestions due to line overloads and, in a market-based system operation, improve the economic followup for the market players [2]. Finally, it is one of the controls, together with topology changes, that fully remain in the hands of Transmission System Operators (TSOs).

Within this perspective, several TSOs, in Europe noticeably, equip their networks with more and more PSTs. Most of them are located to remove congestions on important lines, typically tie-lines between countries, which are usual "bottlenecks" [3].

However, in a large meshed interconnection, PSTs can impact active power flows in far-away distances. As a result, PST control actions taken by one TSO will generally affect the operation of the other TSOs' systems. In some future, these interactions might lead to dangerous conflicting situations, for instance if one TSO prevents parallel power flows from passing through its system. Such "fights" are obviously undesirable, not only from market viewpoint [4], but above all for security of operation.

The optimal solution from a technical viewpoint would probably be a central "entity" coordinating the various PSTs so as to reach a global objective. However, TSOs may not be open to such a solution in which they would partly lose control on equipments that they acquired to improve their own system. Thus, the viewpoint adopted here is more to allow each TSO

The authors are with the Dept. of Electrical Engineering and Computer Science (Montefiore Institute) of the University of Liège, Sart Tilman B37, B-4000 Liège, Belgium. M. Glavic is invited professor and T. Van Cutsem (t.vancutsem@ulg.ac.be) is with the Belgian National Fund for Scientific Research (FNRS) at the same department. to have its own objective, while avoiding conflicts that would endanger security.

To this purpose, this paper first outlines a general framework in which multiple objectives, each relative to a particular TSO, are optimized under a set of common security constraints. While it is assumed that information is shared by the partners in order to avoid violating those constraints, each TSO is supposed to keep its objective undisclosed. The formulation leads to solutions that altogether constitute Nash equilibria of the overall procedure [6]. This general formulation is then particularized to the problem of PST control, for which linear programming is used by each TSO.

\section{MUlti-OBJECTIVE OPTIMIZATION FRAMEWORK}

We consider an environment in which each TSO uses its own controls to optimize an individual objective, all of them operating the same interconnected system. For simplicity, we refer to a case with two TSOs, named TSO1 and TSO2. Let us denote by $\varphi_{1}$ and $\varphi_{2}$, their respective vectors of control variables. The operation of the whole interconnection has to obey a set of inequality constraints:

$$
\mathrm{g}\left(\varphi_{1}, \varphi_{2}\right) \leq \mathbf{0}
$$

which is decomposed according to the involved TSOs, into:

$$
\begin{aligned}
& \mathrm{g}_{1}\left(\varphi_{1}, \varphi_{2}\right) \leq \mathbf{0} \\
& \mathrm{g}_{2}\left(\boldsymbol{\varphi}_{1}, \boldsymbol{\varphi}_{2}\right) \leq \mathbf{0}
\end{aligned}
$$

Each TSO has its own objective function to be minimized. We denote them by $f_{1}\left(\boldsymbol{\varphi}_{1}, \boldsymbol{\varphi}_{2}\right)$ and $f_{2}\left(\boldsymbol{\varphi}_{1}, \boldsymbol{\varphi}_{2}\right)$, respectively. These objectives may be quite different but we assume that each function is influenced by the whole set of controls, which is the expression of the already mentioned TSO interactions. Note that the decision-making procedure may be more complex than just solving a mathematical programming problem: it could be heuristic, or it could involve additional computations, dealing for instance with post-contingency security constraints.

In the worst case, each TSO solves a problem including its own control variables only, the rest being explicitly or implicitly set to some constant value, and focusing on its own operating constraints only. In this perspective, TSO1 computes:

$$
\begin{array}{lc} 
& \boldsymbol{\varphi}_{1}^{*}=\arg \min _{\boldsymbol{\varphi}_{1}} f_{1}\left(\boldsymbol{\varphi}_{1}, \boldsymbol{\varphi}_{2}^{0}\right) \\
\text { subject to } & \mathrm{g}_{1}\left(\boldsymbol{\varphi}_{1}, \boldsymbol{\varphi}_{2}^{0}\right) \leq 0
\end{array}
$$

where $\varphi_{2}^{0}$ is the value of $\varphi_{2}$ assumed by TSO1. A similar set of equations holds for TSO2, which ends up with a solution $\varphi_{2}^{*}$. Since each TSO ignores the other TSO's control 
actions, the operating point resulting from these uncoordinated changes is likely to differ from what each TSO model predicts. More importantly, the solution may not be feasible, since $\mathbf{g}\left(\varphi_{1}^{*}, \varphi_{2}^{*}\right) \leq \mathbf{0}$ does not necessarily hold true.

The more "responsible and coordinated" scheme considered in this paper relies on the following principle:

1) each TSO communicates its current preferred control settings, which are taken into account by the other TSOs;

2) each TSO provides information on its operating constraints;

3) each TSO takes into account the whole set of operating constraints;

4) they iterate until an equilibrium is reached.

Note that the second item may raise confidentiality issues. If this is the case, a consensus has to be reached about the minimal amount of data to be communicated, withholding sensitive pieces of information, so as to render it commercially neutral for instance. On the other hand, a TSO should be able to justify the security constraints it announced (if requested to do so by a regulatory body, for instance).

Thus, at the $k$-th iteration of the procedure, TSO1 knows the current preferred value $\varphi_{2}^{k}$ of TSO2 controls. Using this information, it now computes the updated preferred solution:

$$
\begin{array}{ll} 
& \varphi_{1}^{k+1}=\arg \min _{\boldsymbol{\varphi}_{1}} f_{1}\left(\boldsymbol{\varphi}_{1}, \boldsymbol{\varphi}_{2}^{k}\right) \\
\text { subject to } & \mathrm{g}_{1}\left(\boldsymbol{\varphi}_{1}, \boldsymbol{\varphi}_{2}^{k}\right) \leq 0 \\
& \mathrm{~g}_{2}\left(\boldsymbol{\varphi}_{1}, \boldsymbol{\varphi}_{2}^{k}\right) \leq 0
\end{array}
$$

TSO2 carries out a similar computation, ending up with the updated solution $\varphi_{2}^{k+1}$. Both values are used at the next iteration.

If convergence is achieved, the final solution reached is:

$$
\begin{array}{rr}
\text { for TSO1: } & \boldsymbol{\varphi}_{1}^{*}= \\
\text { subject to } & \arg \min _{\boldsymbol{\varphi}_{1}} f_{1}\left(\boldsymbol{\varphi}_{1}, \boldsymbol{\varphi}_{2}^{*}\right) \\
& \mathrm{g}_{1}\left(\boldsymbol{\varphi}_{1}, \boldsymbol{\varphi}_{2}^{*}\right) \leq 0 \\
& \mathrm{~g}_{2}\left(\boldsymbol{\varphi}_{1}, \boldsymbol{\varphi}_{2}^{*}\right) \leq 0 \\
\text { and for TSO1: } \quad \boldsymbol{\varphi}_{2}^{*}=\arg \min _{\boldsymbol{\varphi}_{2}} f_{2}\left(\boldsymbol{\varphi}_{1}^{*}, \boldsymbol{\varphi}_{2}\right) \\
\text { subject to } \quad & \mathrm{g}_{1}\left(\boldsymbol{\varphi}_{1}^{*}, \boldsymbol{\varphi}_{2}\right) \leq 0 \\
& \mathrm{~g}_{2}\left(\boldsymbol{\varphi}_{1}^{*}, \boldsymbol{\varphi}_{2}\right) \leq 0
\end{array}
$$

where all security constraints are satisfied.

The solution $\left(\varphi_{1}^{*}, \varphi_{2}^{*}\right)$ is a Nash equilibrium of the multiobjective problem. From Game Theory viewpoint, the decisions taken by several players make up altogether a Nash equilibrium if, for each player, there is no advantage in changing his decision, given the decisions taken by the other players [5], [6]. In other words, at a Nash equilibrium, each player chooses a strategy that is the best response to the other players' strategies. In the context of the above problem, each TSO may be viewed as a self-interested player acting in a rational way towards the control actions that optimize its objective, all of them obeying the whole set of operating constraints.

Of course, the convergence of the above procedure and the existence of several Nash equilibria are questions of interest.
Another well-known concept in multi-objective optimization is the one of Pareto optimum. A solution $\varphi^{*}$ is Pareto optimal, if it is feasible and is not dominated by any other feasible solution. A solution $\varphi^{\prime}$ dominates $\varphi^{*}$ if $f_{j}\left(\varphi^{\prime}\right) \leq f_{j}\left(\boldsymbol{\varphi}^{*}\right)$ for all $j$, and $f_{j}\left(\varphi^{\prime}\right)<f_{j}\left(\varphi^{*}\right)$ for at least one $j$. The set of all Pareto optimal solutions is called the Pareto set. By being non-dominated, the members of the Pareto set represent the possible tradeoffs among the various objectives $f_{j}$ [7].

A Nash equilibrium does not necessarily belong to the Pareto set and, vice versa, the members of the Pareto set do not in general constitute equilibria of the above iterative procedure. The Nash equilibrium not being Pareto optimal means that there exist more interesting solutions, yielding better values for all objective functions than the Nash equilibrium [8].

In the two-TSO case, a Pareto optimum can be reached by minimizing with respect to both $\varphi_{1}$ and $\varphi_{2}$ the composite function $w f_{1}+(1-w) f_{2}$, where $0 \leq w \leq 1$. However, this procedure has two drawbacks for the problem of concern. First, there must be an agreement on the value of $w$. This problem is even more complex when there are more than two TSOs. Second, it forces TSOs to disclose their objectives and formulate them so that it enters the composite objective, which is not the case in the approach proposed here.

Anyway, it is an interesting challenge to find a way to move from the Nash equilibrium closer to (ideally on) the Pareto set, especially when the objective functions deal with market aspects, while keeping the objectives undisclosed [8].

\section{APPLICATION TO PST CONTROL PROBLEM}

We consider an environment in which each TSO uses its PSTs to optimize an individual objective. We adopt the DC approximation, which is acceptable for the problem of concern and leads to an insightful linear problem. Future efforts should be directed towards updating the operating constraints when large PST angle excursions take place as well as adjusting the PST impedances with the tap position.

Under the DC approximation, the active power flows in transmission lines can be linearized around a base case operating point, according to:

$$
\mathbf{P}=\mathbf{P}^{0}+\mathbf{S}\left(\varphi-\varphi^{0}\right)
$$

where $\mathbf{P}^{\mathbf{0}}$ is the base case value of active power flows $\mathbf{P}$, and similarly for $\varphi^{0}$ with respect to $\varphi$. The sensitivity matrix $\mathrm{S}$ can be easily derived from the DC (or even AC) load flow equations using a well-known general sensitivity formula. The limits on line power flows take on the form:

$$
-\mathbf{P}^{\max } \leq \mathbf{S}\left(\boldsymbol{\varphi}-\boldsymbol{\varphi}^{0}\right)+\mathbf{P}^{0} \leq \mathbf{P}^{\max }
$$

where $\mathbf{P}^{\max }$ is a vector of maximum branch power flow.

Proceeding with the two-TSO example, these inequalities can be decomposed into:

$$
\begin{aligned}
& -\mathbf{P}_{1}^{\max }-\mathbf{P}_{1}^{0} \leq \mathbf{S}_{11}\left(\boldsymbol{\varphi}_{1}-\varphi_{1}^{0}\right)+\mathbf{S}_{12}\left(\boldsymbol{\varphi}_{2}-\boldsymbol{\varphi}_{2}^{0}\right) \\
& \leq \mathbf{P}_{1}^{\max }-\mathbf{P}_{1}^{0} \\
& -\mathbf{P}_{2}^{\max }-\mathbf{P}_{2}^{0} \leq \mathbf{S}_{21}\left(\boldsymbol{\varphi}_{1}-\boldsymbol{\varphi}_{1}^{0}\right)+\mathbf{S}_{22}\left(\boldsymbol{\varphi}_{2}-\boldsymbol{\varphi}_{2}^{0}\right) \\
& \leq \mathbf{P}_{2}^{\max }-\mathbf{P}_{2}^{0}
\end{aligned}
$$

where the notation is self-explanatory. 
According to what was presented in Section II, the two TSOs will compute a sequence of PST settings according to $(k=1,2, \ldots)$ :

$$
\begin{aligned}
& \text { for TSO1: } \quad \boldsymbol{\varphi}_{1}^{k+1}=\arg \min \varphi_{1} f_{1}\left(\boldsymbol{\varphi}_{1}, \boldsymbol{\varphi}_{2}^{k}\right) \\
& \text { s.t. } \quad-\mathbf{P}_{1}^{\max }-\mathbf{P}_{1}^{0} \leq \mathbf{S}_{11}\left(\boldsymbol{\varphi}_{1}-\boldsymbol{\varphi}_{1}^{0}\right)+\mathbf{S}_{12}\left(\boldsymbol{\varphi}_{2}^{k}-\boldsymbol{\varphi}_{2}^{0}\right) \\
& \leq \mathbf{P}_{1}^{\max }-\mathbf{P}_{1}^{0} \\
& -\mathbf{P}_{2}^{\max }-\mathbf{P}_{2}^{0} \leq \mathbf{S}_{21}\left(\boldsymbol{\varphi}_{1}-\boldsymbol{\varphi}_{1}^{0}\right)+\mathbf{S}_{22}\left(\boldsymbol{\varphi}_{2}^{k}-\boldsymbol{\varphi}_{2}^{0}\right) \\
& \leq \mathbf{P}_{2}^{\max }-\mathbf{P}_{2}^{0} \\
& \varphi_{1}^{\min } \leq \boldsymbol{\varphi}_{1} \leq \boldsymbol{\varphi}_{1}^{\max } \\
& \text { and for TSO2: } \quad \boldsymbol{\varphi}_{2}^{k+1}=\arg \min _{\boldsymbol{\varphi}_{2}} f_{2}\left(\boldsymbol{\varphi}_{1}^{k}, \boldsymbol{\varphi}_{2}\right) \\
& \text { s.t. } \quad-\mathbf{P}_{1}^{\max }-\mathbf{P}_{1}^{0} \leq \mathbf{S}_{11}\left(\varphi_{1}^{k}-\varphi_{1}^{0}\right)+\mathbf{S}_{12}\left(\boldsymbol{\varphi}_{2}-\varphi_{2}^{0}\right) \\
& \leq \mathbf{P}_{1}^{\max }-\mathbf{P}_{1}^{0} \\
& -\mathbf{P}_{2}^{\max }-\mathbf{P}_{2}^{0} \leq \mathbf{S}_{21}\left(\varphi_{1}^{k}-\varphi_{1}^{0}\right)+\mathbf{S}_{22}\left(\boldsymbol{\varphi}_{2}-\boldsymbol{\varphi}_{2}^{0}\right) \\
& \leq \mathbf{P}_{2}^{\max }-\mathbf{P}_{2}^{0} \\
& \varphi_{2}^{\min } \leq \varphi_{2} \leq \varphi_{2}^{\max }
\end{aligned}
$$

where bounds on control variables have been added to line flow constraints.

Several objective functions may be thought of, such as minimum deviation of controls from base case values, minimum active power losses (using an extension of the above DC model), minimum deviation from a desired value of power flowing through a set of lines, etc. Let us repeat that the optimization procedure may be more complex than shown above, the point being that each TSO takes into account the other TSO's controls and the whole set of operating constraints.

To implement the above ideas, information should be exchanged through a network of TSO computers, first to build the model, then to exchange PST setting values until convergence is reached. Before starting the iterations, the load flow Jacobian matrices of each system have to be sent to a central computer, in order to be assembled into a single Jacobian $\mathbf{J}$, subsequently factorized. The $\mathbf{S}$ matrix can be computed column by column; each column requires solving a sparse linear system with $\mathbf{J}$ as matrix of coefficients, and an independent term stemming from the individual TSO systems. Each TSO must also provide the value of its base case and maximum power flows. From there on, optimizations of the type $(13,14)$ are performed independently by the TSOs, with an exchange of the $\varphi_{1}^{k}, \varphi_{2}^{k}$ PST settings in between iterations.

\section{THE PATH TO NASH EQUILIBRIUM}

The iterative procedure suggested in the previous sections may take on the form of:

- either a (computer to computer) negotiation, in which the iterations are performed until reaching an equilibrium, to be the control settings subsequently implemented on the system;

- or an actual step-by-step implementation of the control changes in the course of iterating.

The communication between computers can be synchronous or asynchronous, as sketched in Fig. 1 for a three-TSO case.
In asynchronous operation, each TSO announces its control settings whenever it is ready for, while in a synchronous operation, each TSO is obliged to announce its settings at specific times. Clearly, the synchronous mode yields more ordered operation, in which each TSO calculation remain consistent with the present state of the system. On the contrary, in the asynchronous mode, each TSO performs its calculations based on data referring to different points in time, depending on the moments at which the other TSOs announced their settings. In synchronous operation, if the solution targeted by one TSO is not fully implemented at the time of communicating the settings, the part of it already implemented is communicated.

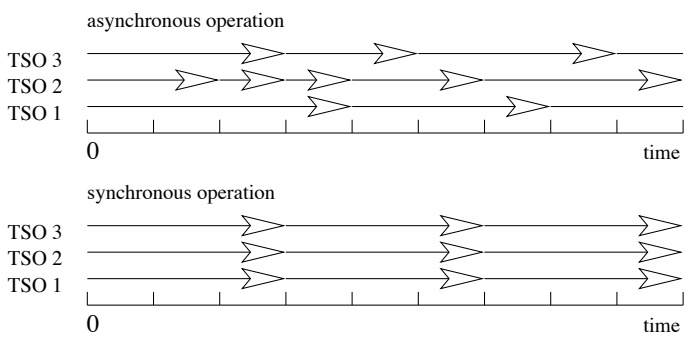

Fig. 1. Asynchronous versus synchronous iterations

Let us further consider the synchronous approach. With reference to the two-TSO case illustrated in Fig. 2, the iterative procedure can be run:

- in a parallel way: TSO1 computes its new settings $\varphi_{1}^{k+1}$ based on the previous setting $\varphi_{2}^{k}$ of TSO2, while in the same time interval TSO2 computes $\varphi_{2}^{k+1}$ based on $\varphi_{1}^{k}$;

- in a sequential way: each TSO waits for the other TSO to communicate its updated settings before performing its own optimization.

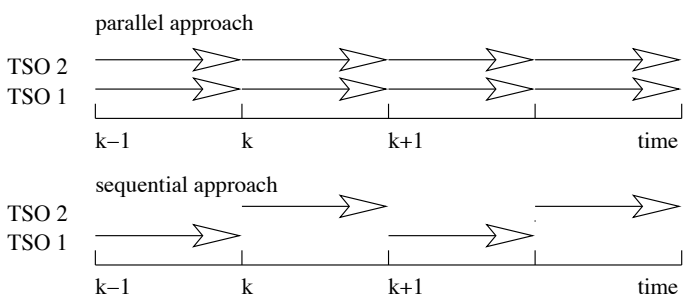

Fig. 2. Parallel versus sequential iterations

The sequential approach has the disadvantage of being slower than the parallel one. This becomes even more important when more than two TSOs are involved, which could be the case in practice. On the other hand, the parallel approach, if applied strictly, may not keep the system inside its feasible region at every moment. Indeed, although the solutions $\left(\varphi_{1}^{k+1}, \varphi_{2}^{k}\right)$ and $\left(\varphi_{1}^{k}, \varphi_{2}^{k+1}\right)$ are both feasible, there is no guarantee that this holds true for the solution $\left(\varphi_{1}^{k+1}, \varphi_{2}^{k+1}\right)$ to be implemented at the next time step. An additional level of coordination is needed to bring the solution back inside the feasible region. This must be designed to avoid oscillating from one side to the other of the feasible region boundary. 


\section{ILLUSTRATIVE EXAMPLE}

\section{A. The System}

We illustrate the proposed method on the well-known IEEE 118-bus test system [9]. The latter has been decomposed into two systems, named respectively "West" and "East" and assumed to be operated by two different TSOs. The overall structure of the so-decomposed system is shown in Fig. 3. Furthermore, a transaction of approximately $240 \mathrm{MW}$ has been added from the Southern part of the East system (where most of its production is located) to the Northern part of the West sytem (where most of its load is located). The largest part of this transaction flows through the Northern part of the East system, thus passing in the so-called "south-north cut" and "north interconnection" (see Fig. 3). This makes the East system operate closer to its limits and with higher losses.

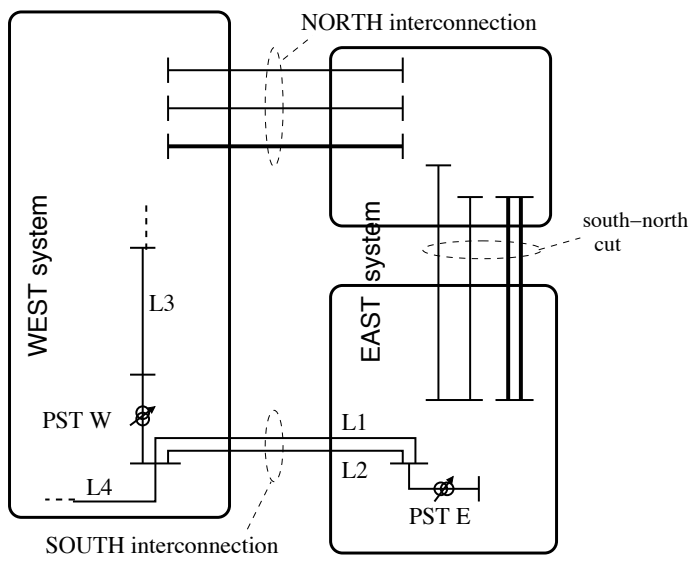

Fig. 3. Overall structure of the decomposed IEEE 118-bus system

Under this perspective, we suppose that TSO East installed a PST in series with tie-lines L1 and L2 of the south interconnection. This allows East to control, up to a certain point dictated by the PST limits, the share of the total power flow between north and south interconnections. However, when more power flows in the south interconnection, line L3 (one of the links between Southern and Northern parts of West system) tends to be overloaded, due to its assumed low thermal capability. Therefore, we assume that TSO West placed a PST in series with that line to protect it.

\section{B. The objectives}

For the above mentioned reasons, the objective of TSO East is to keep below a certain limit the power flowing in the southnorth cut, which is equivalent to keeping above some value the flow in the south interconnection. On the other hand, TSO West wants to keep below a certain limit the power flow in line L3. These two objectives, though not directly connected to each other, turn out to be somewhat in contradiction, in the sense that improving one of them deteriorates the other. This will be shown graphically in the sequel.
C. Examples in the context of step-by-step control implementation

We first present results obtained in the context of a stepby-step implementation of controls by the two TSOs (see beginning of Section IV). Furthermore, we consider the synchronous and sequential schemes. As already discussed, this preserves feasibility of the solution during the iterations. Thus, we assume that each TSO has some time to calculate its next target PST setting, implement a part of it and communicate the resulting new setting to the other TSO.

Figure 4 presents the evolution of the operating point in the control variables space. "phiE" denotes the phase angle of the PST in East and "phiW" the one in West. The two solid lines correspond to the thermal limits of lines L3 and L4. The shaded part of the diagram is the infeasible region. The two dashed dotted lines represent the TSO targets. East has the objective of keeping the active power flow in the south-north cut (see Fig. 3) at $210 \mathrm{MW}$. Points located to the right of that line correspond to higher (undesired) power flows. Similarly, West tries to keep the power flow in line L3 at 30 MW. Clearly, the target line is parallel to the constraint line corresponding to the thermal limit of L3, which has been set to $50 \mathrm{MW}$.

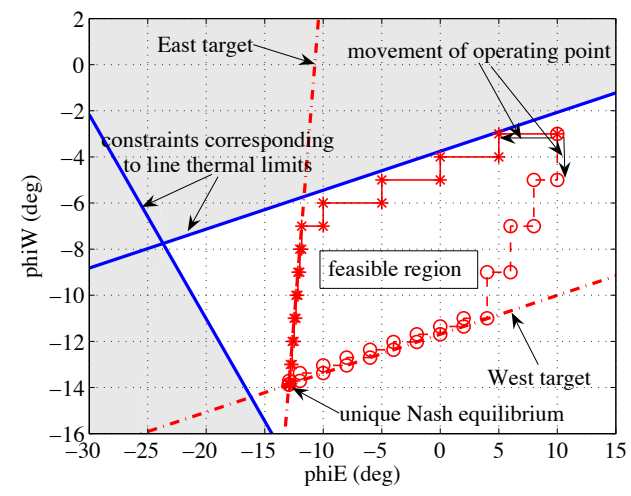

Fig. 4. Convergence to a unique Nash equilibrium

The two trajectories in Fig. 4 correspond to different rates of change of the two PSTs. For the trajectory shown with solid line, it was assumed that, inside the time interval given to announce its new settings, East can change its phase angle by at most 5 degrees, and West by at most 1 degree. The dashed line, on the contrary, corresponds to faster moves by West.

As long as the system operates far enough from constraints, there is a single Nash equilibrium, at the intersection of the target lines in Fig. 4. There, each TSO is satisfied with the solution so it has no motivation to proceed to any change. If the Nash equilibrium point lies inside the feasible region and if this region is convex, the procedure always converges to that point. Changing the relative speeds of the two PSTs does not influence the final point reached.

Remark. Lower limits on PST angle changes must be considered, to avoid moving by less than one step. This has been neglected in Fig. 4 and in subsequent ones, in order not to disturb the discussion with questions regarding discretization. 
Of course, in reality, the procedure will settle down somewhere very close to the aforementioned equilibrium.

In Fig. 5 the target power of East has been decreased to 190 MW. Due to the linearity of the model, this amounts to shifting the target line parallel to itself. As a result, the intersection point of the two target lines does no longer fall in the feasible region. Now the operating point moves along the East target line until it meets the constraint line corresponding to L4 overload. The point cannot move any further since this would either violate the constraint or increase the objective of East TSO. This final point is a Nash equilibrium. Furthermore, all points of the feasibility boundary pointed out in Fig. 5 have the same property and are all Nash equilibria.

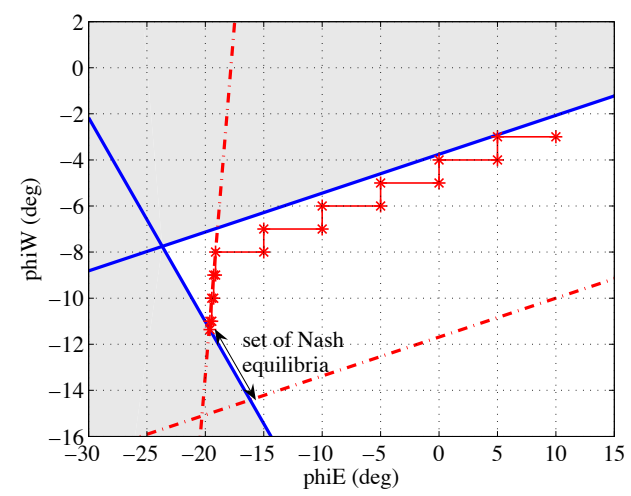

Fig. 5. Target of East decreased to $190 \mathrm{MW}$; multiple Nash equilibria

A similar situation is shown in Fig. 6 corresponding to a $170 \mathrm{MW}$ target power for East. The set of Nash equilibria is larger than in the previous case.

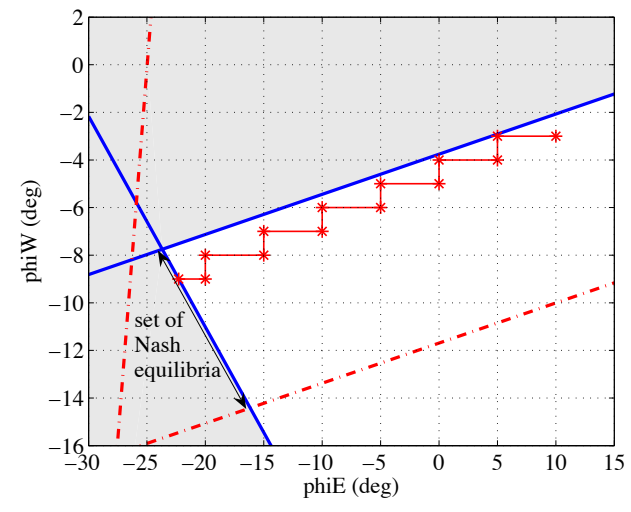

Fig. 6. Target of East decreased to $170 \mathrm{MW}$; multiple Nash equilibria

The final Nash equilibrium reached now depends on the system trajectory, and hence on the starting point and the relative speeds of action of TSOs. As an illustration, consider Fig. 7 which differs from Fig. 6 only by the speeds at which the TSOs change their PST angles (East five times faster than West in Fig. 6, both speeds identical in Fig. 7). A different Nash equilibrium is reached. Moreover, the faster the PST, the better the final value of the corresponding TSO objective.

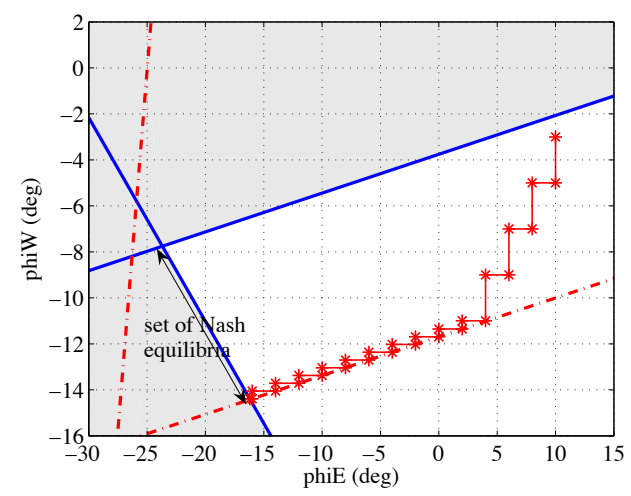

Fig. 7. Same case as in Fig. 6 with different speeds of control changes

Next, we consider in Fig. 8 a simulation starting from an infeasible point, which could result from a disturbance, for instance. According to the algorithm $(13,14)$, the first priority of TSOs is to restore feasibility. Hence, both start taking actions to remove the violation. Note that for TSO East, this action is in a direction opposite to the one dictated by its objective, while there is no such contradiction for TSO West.

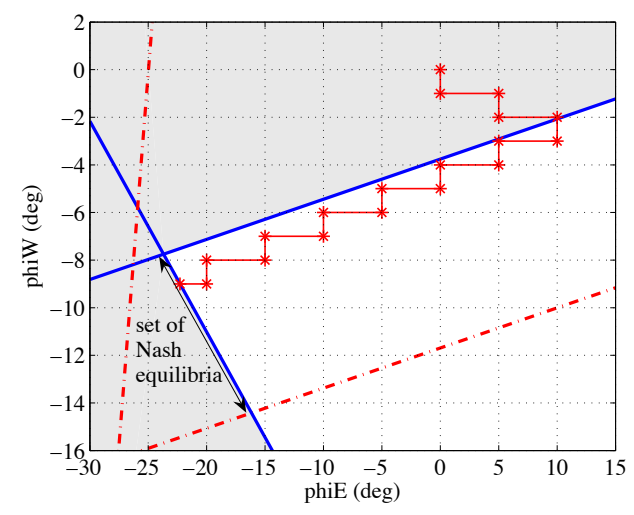

Fig. 8. Sequence starting from an infeasible point

Another view of the same simulation is presented in Fig. 9, showing the successive values of both objective functions. It is easily seen that TSO East has its objective deteriorated until feasibility is restored. From there on, at each iteration, one TSO ameliorates its objective while the other objective is deteriorated. Of course, this deterioration is just a side effect, since a TSO does even not know the other TSO's objective; it only knows its constraints.

It is interesting to note that, in our specific example with linear objective functions, all Nash equilibrium points happen to be Pareto optimal. Indeed, it can be seen from the figures that at those points no combination of PST angle changes can ameliorate both objectives. This would not be the case for another kind of objective functions though.

The sequential scheme considered in the previous examples 


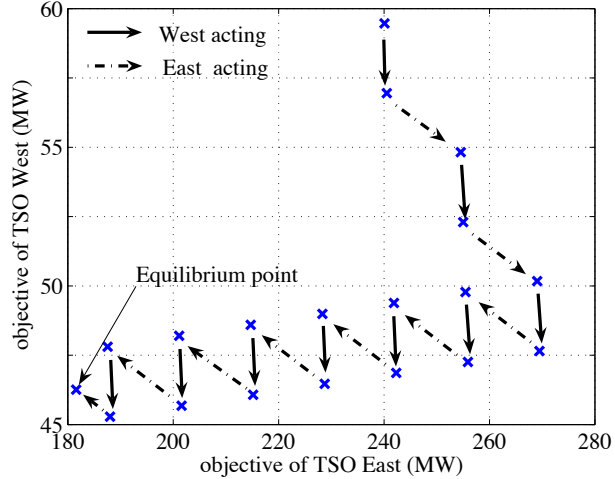

Fig. 9. Case of Fig. 8 seen in the space of objective functions

guarantees that, once inside the feasible region the operating point will always remain inside. Furthermore, Fig. 8 has shown how the procedure can bring the system back inside its feasible region. However, the procedure may not succeed doing so in all cases. An example of difficult situation is depicted in Fig. 10, in which any individual change of the control variables fails bringing the operating point inside the feasible region. In this case, an upper level of coordination is needed, otherwise both TSOs will remain inactive, each one in turn observing its inability to restore feasibility.

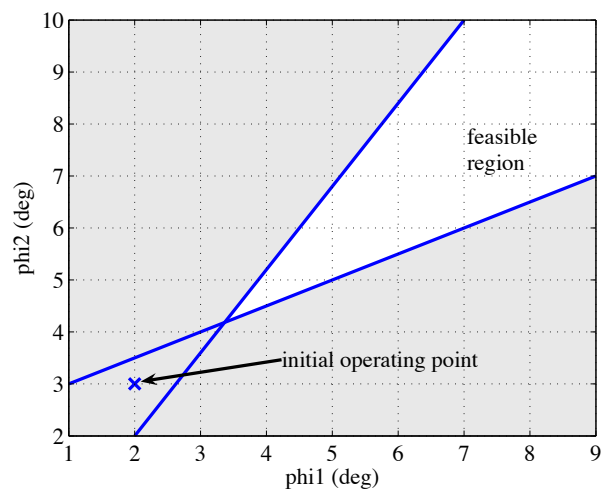

Fig. 10. Initial infeasible point from which the procedure cannot start

\section{Example in the context of negotiation}

Finally, an example of (computer to computer) negotiation (see beginning of Section IV) is given in Fig. 11. Here, the intermediate points do not represent actual operating points, but are rather values announced by each TSO during the iterative procedure. What is sought is the Nash equilibrium, the real PST adjustment taking place later. The scheme is still synchronous and sequential. The control changes are not restricted as in the previous figures, although in the example of Fig. 11, a maximum deviation of 25 degrees has still been imposed. As expected, the convergence to a Nash equilibrium is much faster. Due to the larger steps allowed, the order in which TSOs announce their settings makes a difference on the final equilibrium.

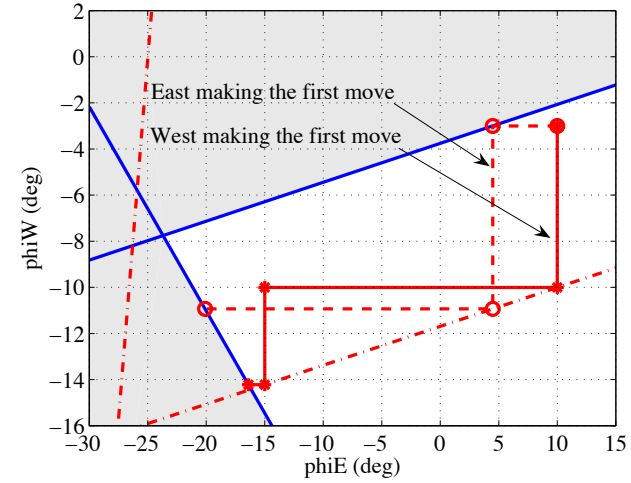

Fig. 11. Example of negotiation

\section{CONCLUSION}

A multi-objective optimisation framework has been proposed to deal with the operation of a system by multiple interacting TSOs. The essence of the algorithm is an iterative approach where TSOs successively compute control actions, taking into account the last actions of other TSOs and obeying the whole set of constraints. This involves information exchange between TSOs, although their individual objectives are kept undisclosed. This framework has been applied to the PST control problem with linearized constraints, and several schemes of potential implementation have been outlined.

Examples relative to a two-PST, two-TSO case have been presented. Several features of the procedure have been illustrated graphically: existence of one or multiple Nash equilibria, sensitivity to relative speeds of action, etc.

Future research should address, among others, the questions of existence and convergence to Nash equilibria, as well as relationships with centralized control and Pareto optimum. In this respect, extensions to controls having a cost and, hence, to market-type objectives are also considered.

\section{REFERENCES}

[1] D. Hayward, J. M. Miller, B. M. Balmat, K. N. Morris, J. H. Malinowski, B. M. Pasternack, L. E. Eilts, "Operating Problems with Parallel Flows", IEEE Trans. on Power Systems, vol. 6, no. 3, 1991, pp. 1024-1034.

[2] B. Marinescu and J.M. Coulondre, "A Coordinated Phase Shifting Control and Remuneration Method for a Zonal Congestion Management Scheme", Proc. Power System Computation and Exposition Conference, New York, Oct. 2004.

[3] P. Bresesti, M. Sforna, V. Allegranza, D. Canever, R. Vailati, "Application of Phase Shifting Transformers for a Secure and Efficient Operation of the Interconnection Corridors", Proc. IEEE PES General Meeting, Denver (Colorado), Paper 001318, 2004.

[4] R. Baldick, E. Kahn "Contract Paths, Phase-Shifters, and Efficient Electricity Trade", IEEE Trans. on Power Systems, vol. 12, no. 2, 1997, pp. 749-755.

[5] T. Basar and G.J. Olsder, Dynamic Noncooperative Game Theory, Academic Press, 1982.

[6] R. Gibbons, "An Introduction to Applicable Game Theory", Journal of Economic Perspectives, vol. 11, no. 1, 1997, pp. 127-149.

[7] S. Talukdar and E. Camponogara, "Network Control as a Distributed, Dynamic Game", Proc. of the 34th Hawaii International Conference on System Sciences, 2001.

[8] P. Correia, T. Overbye and I. Hiskens, "Supergames in electricity markets: beyond the Nash equilibrium conceot", Proc. 14th PSCC, Sevilla, June 2002, session 07, paper 4 .

[9] http://www.ee.washington.edu/research/pstca/ 